\title{
Uso y utilidades clínicas del pletismógrafo en la evaluación de la respuesta sexual masculina:
}

\section{Ejemplificación a partir de tres casos}

\author{
Jesús CASTRO Calvo \\ Dpto. Psicología Básica, Clínica y Psicobiología. Universitat Jaume I \\ castroj@uji.es \\ RAFAEL BALLEster ARNAL \\ Dpto. Psicología Básica, Clínica y Psicobiología. Universitat Jaume I \\ rballest@uji.es \\ MARIA DOLORES GIL LLARIO
} Dpto. Psicología Evolutiva y de la Educación. Universitat de Valencia. Estudi general Dolores. Gil@uv.es

\section{Resumen}

El pletismógrafo peniano es un instrumento que nos permite medir cambios en el diámetro del pene a partir de la exposición a estímulos elicitadores de la respuesta sexual. A pesar de las demostradas utilidades clínicas y empíricas de este instrumento, su uso todavía no se ha generalizado. Por ello, dedicamos este trabajo a ejemplificar el procedimiento a seguir para su aplicación y algunas de las utilidades clínicas que de él se derivan. Para ello recurriremos al análisis de tres casos clínicos; el primero de ellos, un joven con una elevada frecuencia de actividad sexual y de consumo de cibersexo; el segundo, el caso de un chico que a pesar de identificarse como heterosexual, mantiene relaciones sexuales con personas de su mismo sexo: finalmente, analizaremos el caso de otro chico con rasgos de compulsividad sexual. En los tres casos, la aplicación del pletismógrafo complementa una evaluación clásica de la conducta sexual y supone un elemento crucial en el análisis de cada caso.

Palabras clave: Pletismógrafo peniano, Respuesta sexual masculina, Compulsividad sexual, Análisis de casos, Jóvenes

1. Este trabajo ha sido financiado por el proyecto de investigación del Ministerio de Ciencia e Innovación (PSI2011-27992 / 11 I 384). 


\begin{abstract}
The penile plethysmograph is an instrument for measuring changes in the diameter of the penis after exposure to stimuli elicitors of sexual response. Despite the demonstrated clinical and empirical utility of this instrument, its use is not yet widespread. For this reason, we dedicate this paper to illustrate the procedure for application and some of its clinical utilities. For this purpose, we analyze three cases; the first case, a young man with a high frequency of sexual activity and consumption of cybersex; the second, the case of a boy who self-identifies as heterosexual but has relations with same sex people; finally, we discuss the case of another boy with sexual compulsivity traits. In all three cases, the penile plethysmograph complements classical assessment of sexual behavior and it's a crucial element in the analysis of each case.
\end{abstract}

Keywords: Penile plethysmograph, Male sexual response, Sexual compulsivity, Case analysis, Youth

\title{
Introducción
}

El pletismógrafo peniano es un instrumento que nos permite medir cambios en el diámetro del pene a partir de la exposición a estímulos elicitadores de la respuesta sexual. Las primeras versiones de esta herramienta se remontan a 1908 (Freund, 1981), cuando se comenzó a utilizar como medio para medir el efecto de ciertas sustancias sobre el sistema vasomotor de reflejos. Estas primeras aplicaciones sobre todo en el campo de la medicina han ido dando paso a la búsqueda de su utilidad en el ámbito de la atención sexual, disciplina en la que precisamente el pletismógrafo ha hecho mayores contribuciones. La primera línea de investigación que se benefició de esta herramienta fue la del estudio de preferencias. Freund (1963) concluyó tras una investigación con varones heterosexuales y homosexuales que el pletismógrafo era una herramienta válida para discriminar la orientación sexual de las personas, aun cuando ésta se intentara enmascarar voluntariamente. Más allá de las preferencias por un sexo concreto, el pletismógrafo también se ha utilizado para evaluar los contenidos sexuales más habituales hacia los que hombres y mujeres suelen sentir atracción sexual (Rubinsky, Eckerman, Rubinsky y Hoover, 1987).

Otra de las líneas de investigación donde el pletismógrafo ha jugado un papel importante es en la investigación básica y aplicada en trastornos sexuales. Gracias al pletismógrafo peniano se pudo comprender con un poco más de precisión el papel que el estado psicológico de una persona jugaba en problemas de disfunción eréctil (Sakheim, Barlow, Abrahamson y Beck, 1987). En su vertiente más aplicada, el pletismógrafo también se ha utilizado como referencia acerca de la eficacia del tratamiento en patologías sexuales (Herman y Presitt, 1974) o como tratamiento mismo, mejorando el control voluntario sobre la erección por medio de autoinstrucciones y retroalimentación sobre la ejecución (Rosen, Shapiro y Schwartz, 1975).

Finalmente, una de las líneas de investigación que más se ha beneficiado de esta herramienta es el estudio de las parafilias o desviaciones sexuales. Son muchos los estudios que enumeran las bondades del uso de esta metodología en este ámbito (para una revisión a fondo de la aplicación del pletismógrafo en parafilias, Laws y O'Donohue, 2008). El principal escollo en la evaluación de las parafilias por medio de los instrumentos clásicos (entrevistas, autoinformes, escalas, etc.) es que en muchos casos, los pacientes tienen un notable interés 
por enmascarar sus intereses sexuales (o bien porque están siendo investigados por posibles delitos sexuales o simplemente por la vergüenza que les supone esta situación), por lo que este tipo de medidas no suelen ser ni fiables ni válidas. Por el contrario, el pletismógrafo peniano es una medida objetiva de la respuesta sexual, difícilmente modificable por mucho interés que el evaluado tenga en ello. Aprovechándose de esta ventaja, el pletismógrafo se ha utilizado como medio fiable y valido de evaluación de distintas parafilias (sobre todo pedofilia, hebefilia o sadismo sexual), como criterio para evaluar los beneficios de una intervención, como medida para calcular el riesgo de reincidencia y englobando todas las anteriores, como prueba forense en casos de delitos contra la libertad sexual de las personas.

A pesar de que como hemos visto, existen multitud de campos donde el uso del pletismógrafo puede resultar útil, su uso prácticamente no se ha extendido. La mayoría de las investigaciones utilizando esta metodología se han realizado en EEUU, donde el pletismógrafo tiene la consideración de prueba clínica y pericial y está regulada como cualquier otro procedimiento médico. En España, no nos consta que ningún equipo de investigación o institución esté haciendo uso de esta metodología. Existe un libro publicado por el profesor José Cáceres (2001) donde se describe con detalle la metodología de aplicación y sus utilidades clínicas, pero donde no se precisa si el autor lo ha aplicado o con qué objetivos.

En nuestro caso, el uso del pletismógafo peniano tiene por objeto integrar las distintas aportaciones de cada una de las anteriores líneas de investigación bajo un marco comprensivo más general, la compulsividad sexual. La compulsividad sexual (cs) es una dimensión que permite dar respuesta a la pregunta de hasta qué punto una persona es capaz de controlar sus impulsos sexuales. A nivel teórico, tres evidencias fundamentan la aplicación del pletismógrafo en el estudio de la compulsividad sexual. La primera es que se ha encontrado una comorbilidad de hasta el 33 \% entre diagnósticos de cS y de otras parafilias (Krueger, Kaplan y First, 2009). La segunda se fundamenta en el hallazgo de una íntima relación entre cs y erotofilia (Byers, Purdon y Clark, 1998), dimensión que refleja la tendencia a responder mediante activación sexual a cualquier tipo de estímulo sexual. Si a las dos evidencias anteriores le sumamos el hecho de que uno de los elementos centrales en el diagnóstico de la cs es la incapacidad para controlar voluntariamente la respuesta sexual (Kafka, 2010), entonces no cabe duda de que el pletismógrafo se dibuja como una herramienta muy eficaz a la hora de complementar otro tipo de instrumentos diagnósticos en la evaluación de la CS.

El objetivo de este artículo es exponer la metodología que seguimos para la medida de la respuesta sexual en el hombre y ejemplificar alguna de sus utilidades clínicas por medio del análisis de tres casos de chicos jóvenes que acudieron voluntariamente para someterse a una evaluación completa de su conducta sexual.

\section{Método}

\section{Participantes}

Para ejemplificar las aplicaciones clínicas del pletismógrafo se han seleccionado tres casos de chicos jóvenes en los que, por sus características, resultaba interesante la aplicación del pletismógrafo. Los tres fueron evaluados en los laboratorios de nuestra Unidad de Investigación sobre Sexualidad y Sida (UNISEXSIDA) en la Universitat Jaume I. En la sección de resultados se presentan con detalle las características demográficas y clínicas de los tres participantes. 


\section{Instrumentos}

Los participantes fueron sometidos a una extensa evaluación donde se aplicaron gran variedad de instrumentos que se pueden clasificar en las siguientes dimensiones:

1. Compulsividad sexual: evaluada mediante tres instrumentos de screening breves: la Escala de Compulsividad Sexual (SCS), el Inventario de Hipersexualidad (IH) y el Cuestionario de Adicción al Sexo (SAST).

2. Variables clínicas: psicopatología en eje I y II evaluada mediante la entrevista clínica estructurada para los trastornos del eje I del DSM-IV (SCID-I) y del eje II (SCID-II). También se aplicaron cuestionarios para evaluar depresión (BDI-II), ansiedad estado/rasgo (STAI) y autoestima (RSEI).

3. Conducta sexual: mediante la escala de búsqueda de sensaciones sexuales (BSS) se midió la propensión a mantener relaciones sexuales novedosas. Además se registró el consumo de cibersexo tanto voluntario (ISST) como involuntario (instrumento diseñado ad-hoc) y otros aspectos más generales de la conducta sexual como la frecuencia de actividad sexual, el tipo de prácticas realizadas, número de parejas sexuales, etc. (Encuesta sobre el Sida, ENSI).

4. Medida fisiológica de la respuesta sexual en varones: se utilizó como herramienta de registro el pletismógrafo peniano (modelo Monarch 21). Este instrumento permite recoger tres tipos de respuesta fisiológica (respuesta electrodérmica, amplitud de la respiración y diámetro del pene) ante la presentación de 22 segmentos de estímulos (en la gráfica 1 se enumera el contenido de cada segmento).

Cada segmento comienza con una imagen sugerente (únicamente se exponen imágenes sexuales explícitas de mujeres adultas en dos segmentos) de la persona o situación sobre la que va la historia. Tras diez segundos, esta imagen desaparece y una voz masculina narra durante 85 segundos una historia de contenido sexual relacionada con la imagen del principio. Esta historia puede ser de sexo consentido o forzado (coercitivo). Cuando finaliza la narración oral se exponen 4 imágenes más similares a las del inicio de la situación y en caso de experimentarse una erección, se da el tiempo necesario para que el pene vuelva a su estado basal. En la figura 1 se puede ver una situación habitual de evaluación.

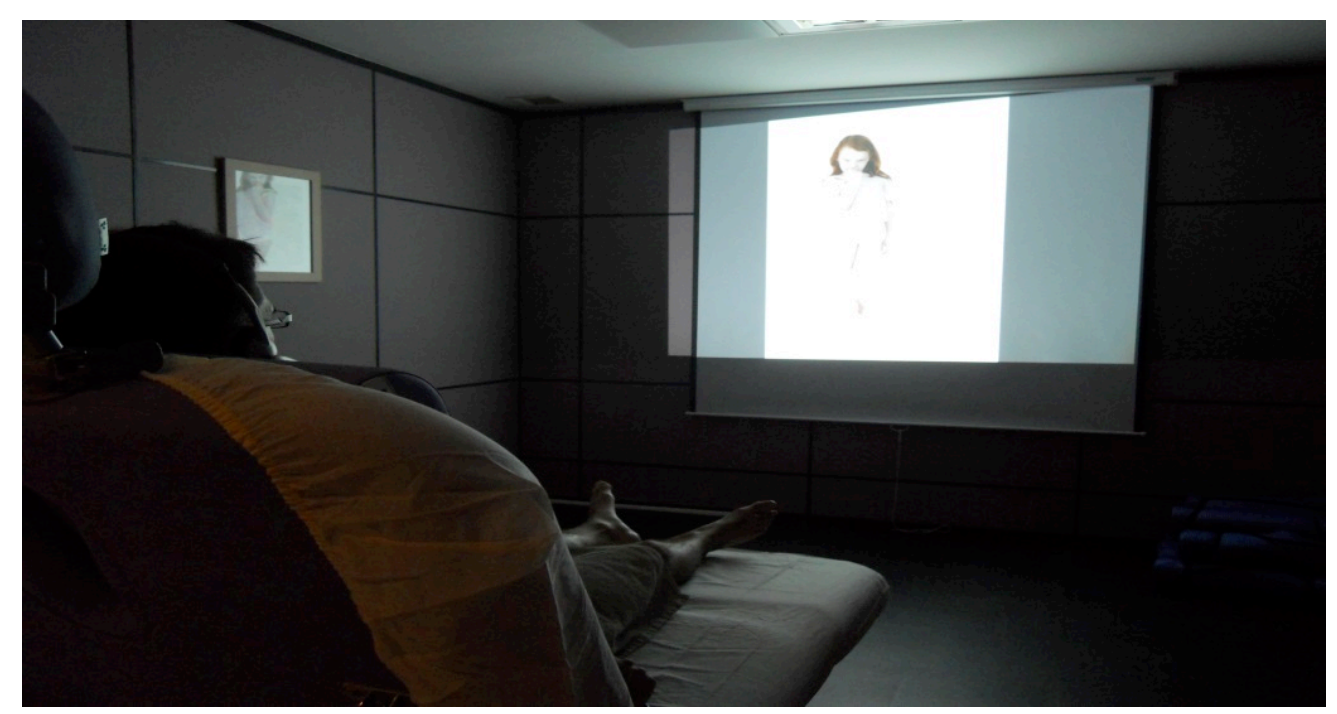

Figura 1. Situación habitual de evaluación 


\section{Procedimiento}

La investigación mediante el pletismógrafo constituye una de las fases dentro de una investigación más amplia sobre control de los impulsos sexuales. Esta investigación, financiada por el Ministerio de Ciencia e Innovación, se desarrolla en tres fases. Actualmente estamos finalizando la segunda fase del proyecto.

En la primera fase, se evaluó a un amplio grupo de jóvenes (exactamente a 1316) mediante los tres instrumentos de screening de la compulsividad sexual antes mencionados. De esos 1316, seleccionamos para la segunda fase de esta investigación a 100 chicos y 100 chicas con puntuaciones clínicas en estos cuestionarios (grupo experimental) y a otros tantos con puntuaciones normales (grupo control). Para aumentar el número de participantes experimentales distribuimos por las distintas facultades de la Universitat Jaume I de Castellón carteles con una breve descripción de los principales síntomas de la compulsividad sexual y nuestros datos de contacto (por si alguna otra persona se sentía identificada y deseaba contactar).

En la segunda fase, concertábamos una cita individual en la que a todos ellos se les realizaba la entrevista clínica y el pase de cuestionarios (unas 2 horas de duración). Su participación se remuneraba con 10 euros. A los chicos se les ofrecía la posibilidad de seguir participando mediante la aplicación del pletismógrafo, remunerada con 10 euros adicionales. A los que aceptaban, se les facilitaba una cinta métrica de precisión para la medida del diámetro del pene (información necesaria para la selección del correcto anillo peniano) y se concertaba una nueva cita, donde finalmente se realizaba la prueba. La duración de la aplicación del pletismógrafo es de alrededor de 2 horas.

\section{Análisis de datos}

Dado que en este trabajo se ha seguido una metodología experimental de estudio de caso, la estrategia de análisis de los resultados que hemos decidido aplicar ha sido de análisis visual. El análisis visual consiste en la interpretación de los resultados sin recurrir a ningún contraste estadístico, únicamente buscando la significación clínica para el paciente concreto y recurriendo en todo caso a la comparación con los baremos establecidos para cada escala. Es la técnica de análisis más utilizada en este tipo de diseños experimentales (Arnau, 1995) donde la significación clínica es más importante que la estadística.

\section{Resultados}

La gráfica 1 representa la diferencia en centímetros entre el pene en estado basal y el grado de erección máximo alcanzado durante cada segmento. La línea roja vertical $(0,70 \mathrm{~cm})$ representa el valor a partir del cual se considera que el aumento del diámetro del pene es significativo, si bien una erección total puede llegar a alcanzar una diferencia de hasta $4 \mathrm{~cm}$. con el pene en estado de flacidez.

\section{Caso 1}

El caso 1 corresponde a un chico de 23 años, heterosexual y con pareja estable. La entrevista clínica no reveló psicopatología significativa ni en la actualidad ni a lo largo de su 
vida y su puntuación en el resto de escalas clínicas se encontraba dentro de la normalidad. Al evaluar su conducta sexual, llama la atención la alta frecuencia de orgasmos semanales que mantiene (entre 12 y 13), tanto por masturbación (sobre todo visionando pornografía) como manteniendo relaciones con su pareja. El evaluado dedica alrededor de 7 horas semanales a la práctica de cibersexo. Sin embargo, sus puntuaciones en las distintas escalas de compulsividad sexual no denotan signos de pérdida de control sobre sus impulsos sexuales.

Respecto a los resultados en el pletismógrafo, si nos fijamos en la gráfica 1 este chico únicamente muestra cierta excitación ante los dos segmentos en los que se evocan situaciones sexuales con mujeres adultas (mujeres adultas persuasivo y mujeres explícito 1). En ambos casos, el nivel de activación sexual, traducido en un aumento del diámetro del pene de 0.7-0.9 cm., no podría considerarse de magnitud suficiente como para clasificarlo como erección completa.

Mujeres adultas persuasivo
Mujeres adultas coercitivo
Chica adolescente persuasivo
Chica adolescente coercitivo
Chica escolar persuasivo
Chica escolar coercitivo
Chica pre-escolar persuasivo
Chica pre-escolar coercitivo
Hombre adulto persuasivo
Hombre adulto coercitivo
Chico adolescente persuasivo
Chico adolescente coercitivo
Chico escolar persuasivo
Chico escolar coercitivo
Chico pre-escolar persuasivo
Chico pre-escolar coercitivo
Neutral
Cibersexo con una chica
Pornografía en Internet
Mujeres explícito 1
Mujeres explícito 2

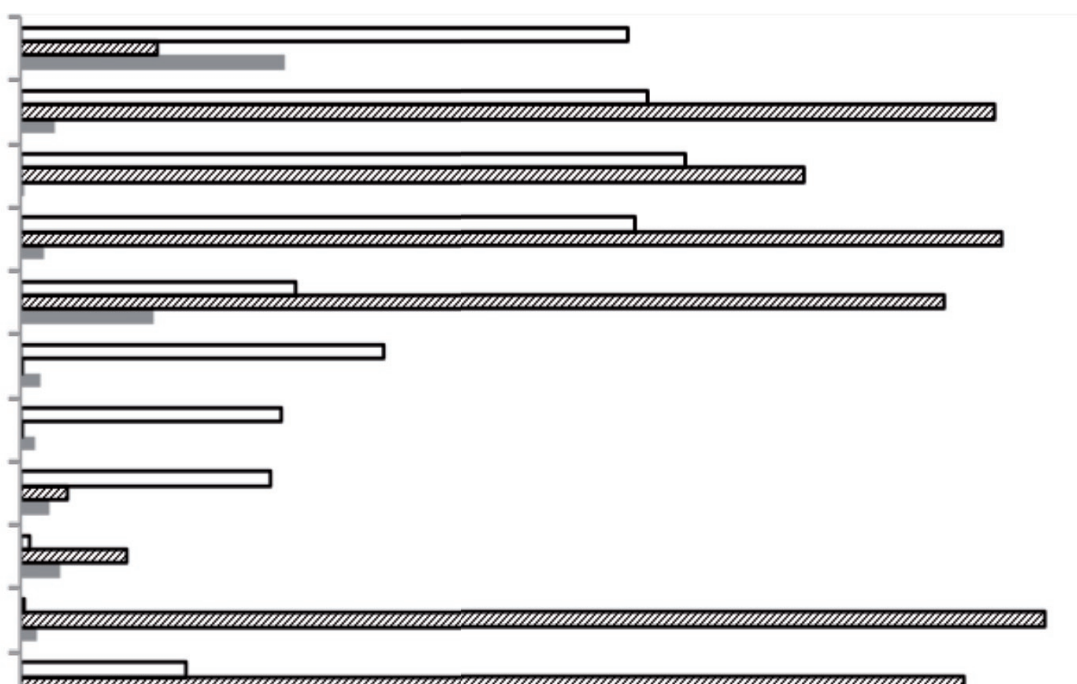

Chico adolescente persuasivo

Chico escolar persuasivo
Chico escolar coercitivo
Chico pre-escolar persuasivo
Chico pre-escolar coercitivo
Neutral
Hombres explícito
Mujeres explícito 1
Mujeres explícito 2
Cibersexo con una chica
Pornografía en Internet
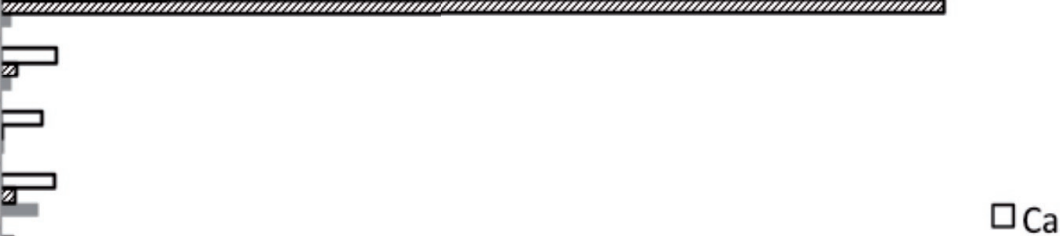

$\square$ Caso 3

$$
\text { 赵 }
$$

Caso 2

Caso 1

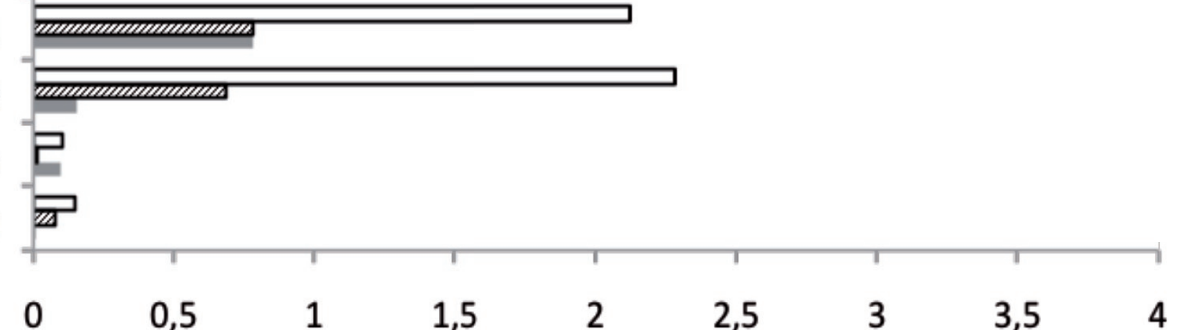

Gráfica 1. Respuesta sexual por cada segmento 


\section{Caso 2}

En el caso 2 nos encontramos con un chico de 21 años sin pareja estable. De nuevo, tanto la entrevista como el resto de escalas clínicas no revelan psicopatología en Eje I y Eje II. Tampoco encontramos nada reseñable en su conducta sexual excepto cuando evaluamos su orientación sexual: a pesar de que señala ser heterosexual, sentirse atraído únicamente hacia el otro sexo y no desear ni fantasear con personas de su mismo sexo, sí ha mantenido relaciones sexuales con chicos.

En este caso, la aplicación del pletismógrafo nos permite constatar una alta reactividad sexual ante una gran variedad de estímulos femeninos pero también hacia algunos masculinos. Como se aprecia en la gráfica 1, el evaluado muestra una alta activación sexual (aumento de hasta $3,5 \mathrm{~cm}$. en el diámetro del pene) ante 4 de los segmentos femeninos (mujeres adultas coercitivo, chicas adolescentes coercitivo y persuasivo y chicas escolares persuasivo) y una activación ligeramente mayor (de hasta $3,6 \mathrm{~cm}$.) ante dos segmentos masculinos (hombre adulto coercitivo y chico adolescente persuasivo).

\section{Caso 3}

El caso 3 corresponde a un varón de 19 años con pareja estable. Como en los otros dos casos, no presenta patología psicológica ni en la actualidad ni a lo largo de la vida. Al explorar su conducta sexual, encontramos una frecuencia sexual semanal un tanto superior a la media para su edad y sexo (5 orgasmos semanales) y un consumo de cibersexo también ligeramente mayor (3 horas semanales), si bien se puede considerar todavía dentro de un rango normal. Las puntuaciones en compulsividad sexual, a pesar de no alcanzar el criterio para considerarse clínicas, si son bastante altas (percentil 70).

La aplicación del pletismógrafo (gráfica 1) nos permite observar una clara preferencia sexual hacia las chicas, sobre todo adultas (mujeres adultas persuasivo y coercitivo) y adolescentes (chicas adolescentes persuasivo y coercitivo). En estos segmentos, el diámetro del pene del evaluado aumenta entre 2,1 y $2,4 \mathrm{~cm}$. con respecto al estado basal. También puede observarse cierta activación sexual en segmentos de chicas de edades menores (chicas escolar y pre-escolar persuasivo y coercitivo), si bien esta respuesta sexual es menos intensa que en los otros casos (entre 0,9 y 1,4 cm. de aumento del diámetro del pene).

\section{Conclusiones}

Los casos analizados dan buena cuenta de algunas de las utilidades clínicas derivadas del uso del pletismógrafo. En todos ellos, el pletismógrafo peniano se ha mostrado eficaz como complemento para enriquecer los hallazgos encontrados en la evaluación clásica de la conducta sexual.

En el primer caso, la alta frecuencia sexual y de consumo de cibersexo nos hacía pensar en una incipiente pérdida del control sobre la conducta sexual, a pesar de que los resultados en los cuestionarios que miden esta dimensión eran normales. Ante esta discrepancia, el uso de esta metodología objetiva nos podría permitir averiguar si el paciente había tratado de enmascarar ciertos problemas en el control de los impulsos sexuales contestando favorablemente en las distintas escalas. De ser así, en la evaluación de su respuesta sexual deberíamos observar un patrón de activación sexual muy significativo ante gran variedad de estímulos elicitadores de la respuesta sexual (en el caso de este varón heterosexual, ante segmentos de 
chicas o bien segmentos que recrean interacciones típicas del cibersexo). Sin embargo, esta idea queda rechazada cuando tras la aplicación del pletismógrafo comprobamos que sus resultados se adaptan a la respuesta sexual esperable en un varón normal (ligera excitación ante estímulos de mujeres adultas).

Del análisis de este caso se desprende además una idea muy comentada en la literatura sobre compulsividad sexual: no debemos confundir una alta frecuencia de actividad sexual con una pérdida del control sobre la misma (Kafka, 2008). Son muchas las personas que durante épocas de su vida, por muy distintos motivos (p.e., porque inician una nueva relación, porque acaban de quedar solteros y gustan de conocer a nuevas parejas sexuales, etc.), pueden mantener una alta frecuencia de actividad sexual sin que esto suponga problema alguno en sus vidas. Por ello, el uso del pletismógrafo puede resultar de especial utilidad en casos donde la información obtenida mediante distintas escalas resulta contradictoria o confusa.

El segundo caso representa un buen ejemplo de otra de las principales utilidades del pletismógrafo. Estudios clásicos demostraron la utilidad de esta herramienta a la hora de determinar la orientación sexual, aun cuando existía voluntad por enmascararla (Freund, 1963). En este segundo caso, el pletismógrafo se dibuja como técnica eficaz a la hora de aclarar las discrepancias entre cómo el paciente define su orientación sexual y ciertas conductas sexuales que ha realizado y contradicen esta identificación: este paciente se definía como heterosexual y refería no sentir atracción o deseo alguno hacia personas de su mismo sexo, si bien sí afirmaba haber mantenido relaciones homosexuales. La aplicación del pletismógrafo nos permite comprobar cómo el paciente muestra importantes niveles de activación sexual ante estímulos de ambos sexos, patrón de respuesta sexual que corresponde a una orientación bisexual. Una vez confirmada la atracción sexual hacia ambos sexos, podemos pensar que las reticencias por identificarse con una orientación sexual bisexual pueden deberse o bien a que el evaluado no se sentía lo suficientemente cómodo para identificarse como bisexual durante la evaluación o a que experimenta cierta homofobia interiorizada que le impide asumir algunos aspectos de su sexualidad.

En el último caso, las puntuaciones algo elevadas en los cuestionarios de compulsividad sexual (percentil 70) unido a que también mostraba una frecuencia de actividad sexual y de consumo de cibersexo superiores a la media hacían interesante la aplicación del pletismógrafo. En este caso, la evaluación de la respuesta sexual constató una activación sexual significativa al explorar escenas relacionadas con atracción sexual sobre todo hacia mujeres adultas, pero también una activación sexual significativa ante chicas de edades mucho menores.

Una interpretación prematura podría llevarnos a pensar que nos encontramos ante un patrón de respuesta sexual característico de una persona con tendencias parafílicas o con mayor probabilidad de cometer una agresión sexual. Sin embargo, este perfil no se adapta al patrón clásico de un paciente pedófilo o agresor sexual. En estos casos, el patrón de activación sexual suele ser precisamente a la inversa: una mayor activación sexual en segmentos de niñas de edades más tempranas y una menor intensidad de la respuesta sexual conforme aumenta la edad (Barsetti, Earls, Lalumiere y Belanger, 1998; Grossman, Cavanaugh y Haywood, 1992). Que el evaluado haya mostrado activación sexual significativa ante chicas de corta edad probablemente se deba más a esa tendencia de personas con cierto grado de compulsividad sexual a responder eróticamente a cualquier situación que trate de despertar su deseo sexual que al propio contenido que la desencadena (Byers, Purdon y Clark, 1998). 


\section{Referencias}

Arnau, J. (1995). Análisis estadístico de datos para los diseños de sujeto único. En M. T. Anguera, J. Arnau, M. Ato, R. Martínez, J. Pascual y G. Vallejo (eds.), Métodos de investigación en psicología (pp. 179-193). Madrid: Síntesis.

Barsetti, I., Earls, C. M., Lalumiere, M. L. y Belanger, N. (1998). The differentiation of intrafamilial and extrafamilial heterosexual child molesters. Journal of Interpersonal Violence, 13, 275 - 286.

Byers, E. S., Purdon, C. y Clark, D. A. (1998). Sexual intrusive thoughts of college students. The Journal of Sex Research, 35 (4), 359-369.

Cáceres, J. (2001). Sexualidad humana: diagnóstico psicofisiológico. Bilbao: Universidad de Deusto.

Freund, K. (1963). A laboratory method for diagnosing predominance of homosexual or hetero erotic interest in the male. Behavior Research Therapy, 1, 85-93.

Freund, K. (1981). Assessment of pedophilia. En M. Cook y K. Howells (eds.), Adult sexual interest in children. New York: Academic Press.

Grossman, L. S., Cavanaugh, J. L., y Haywood, T. W. (1992). Deviant sexual responsiveness on penile plethysmography using visual stimuli: Alleged child molesters vs. normal control subjects. Journal of Nervous and Mental Disease, 180, 207-208.

Herman, S. H. y Presitt, M. (1974). An experimental analysis of feedback to increase sexual arousal in a case of homo and heterosexual impotence. Journal of Behavior Therapy and Experimental Psychiatry, 5, 271-274.

Kafka, M. P. (2008). Neurobiological processes and comorbidity in sexual deviance. En D. R. Laws, y W. T. O’Donohue (Edits.), Sexual Deviance (Second edition) (págs. 571-593). New York: The Guildford Press.

Kafka, M. P. (2010). Hypersexual Disorder: A proposed Diagnosis for DSM-V. Archives of Sexual Behavior, 39(2), 377-400.

Krueger, R. B., Kaplan, M. S. y First, M. B. (2009). Sexual and other Axis 1 diagnoses of 60 males arrested for crimes against children involving the Internet. CNS Spectrums. 14, 623-631.

Laws, D. R. y O'Donohue, W. T. (2008). Sexual deviance: Theory, assessment, and treatment. New York: The Guilford Press.

Rosen, R. C., Shapiro, D. y Schwartz, G. E. (1975). Voluntary control of penile tumescence. Psychosomatic Medicine, 37, 479-483.

Rubinsky, H., Eckerman, D., Rubinsky, E. y Hoover, C. (1987). Earlyphase psychological response patterns to psychosexual stimuli: comparison of males and females patterns. Archives of Sexual Behavior, 19, 45-56.

Sakheim, D. K., Barlow, D. H., Abrahamson, D. J. y Beck, J. G. (1987). Distinguishing between organogenic and psychogenic erectile dysfunction. Behavior Research Therapy, 25 (5), 379-390. 